\title{
Cost-Effectiveness of Brexanolone Versus Selective Serotonin Reuptake Inhibitors for the Treatment of Postpartum Depression in the United States
}

\author{
Adi Eldar-Lissai, PhD; Joshua T. Cohen, PhD; Samantha Meltzer-Brody, MD, MPH; \\ Margaret E. Gerbasi, PhD; Elizabeth Chertavian, MBA; Paul Hodgkins, PhD; \\ Julia C. Bond, MPH; and Scott J. Johnson, PhD, MHA
}

\begin{abstract}
BACKGROUND: Brexanolone injection (BRX) was approved by the FDA in 2019 for the treatment of adult patients with postpartum depression (PPD), but its cost-effectiveness has not yet been evaluated.

OBJECTIVE: To estimate the cost-effectiveness of BRX compared with treatment with selective serotonin reuptake inhibitors (SSRIs) for PPD.

METHODS: We projected costs (2018 U.S. dollars) and health (qualityadjusted life-years [QALYS]) for mothers treated with BRX or SSRIs and their children. A health state transition model projected clinical and economic outcomes for mothers based on the Edinburgh Postnatal Depression Scale, from a U.S. payer perspective. The modeled population consisted of adult patients with moderate to severe PPD, similar to BRX clinical trial patients. Short-term efficacy for BRX and SSRIs came from an indirect treatment comparison. Long-term efficacy outcomes over 4 weeks, 11 years (base case), and 18 years were based on results from an 18-year longitudinal study. Maternal health utility values came from analysis of trial-based short-form $6 \mathrm{D}$ responses. Other inputs were derived from the literature.

RESULTS: The incremental cost-effectiveness ratio for BRX versus SSRIs was $\$ 106,662$ per QALY gained over an 11-year time horizon. Drug and administration costs for BRX averaged \$38,501, compared with \$25 for SSRIs over the studied time horizon. Maternal total direct medical costs averaged $\$ 65,908$ in the BRX arm, compared with $\$ 73,653$ in the SSRI arm. BRX-treated women averaged 6.230 QALYs compared with 5.979 QALYs for the SSRI arm. Adding partner costs and utilities in a sensitivity analysis further favored BRX. Results were sensitive to the severity of PPD at baseline and the model time horizon. Probabilistic sensitivity analyses indicated that BRX was cost-effective at the $\$ 150,000$-per-QALY threshold with $58 \%$ probability.
\end{abstract}

CONCLUSIONS: Analysis using a state transition model showed BRX to be a cost-effective therapy compared with SSRIs for treating women with PPD.

J Manag Care Spec Pharm. 2020;26(5):627-38

Copyright @ 2020, Academy of Managed Care Pharmacy. All rights reserved.

\section{What is already known about this subject}

Postpartum depression (PPD) affects an estimated $11.5 \%$ of mothers in the United States and can have significant and long-lasting detrimental effects on mothers, children, and families.

PPD has historically been treated with antidepressants, such as selective serotonin reuptake inhibitors (SSRIs) alone or in conjunction with low-intensity interventions; however, these pharmacological treatments are not specifically indicated for PPD and have not been evaluated in robust clinical trials for this indication.

In 2019, brexanolone injection (BRX) became the first treatment approved by the FDA for adult patients with PPD, based on the positive efficacy, safety, and tolerability profile demonstrated in 2 phase 3 clinical trials.

\section{What this study adds}

This study models comparative clinical and economic outcomes for BRX compared with SSRIs in mothers with PPD and their children and represents the first economic model of pharmacotherapy for the treatment of PPD.

The relative cost-effectiveness of BRX depends on the assumed efficacy of SSRIs, depressive severity at baseline, and duration of model time horizon.

Treatment of PPD with BRX is likely to be cost-effective relative to SSRIs from a U.S. payer perspective.

D ostpartum depression (PPD) is one of the most common medical complications during and after pregnancy, affecting an estimated $11.5 \%$ of mothers in the United States. ${ }^{1-7}$ Symptoms of PPD include depressed mood, diminished ability to concentrate and perform usual tasks, and decreased maternal-infant interaction. ${ }^{8,9}$ PPD can also lead to suicidality, with suicide being a leading cause of pregnancyrelated mortality; some studies estimate that nearly $1 \%$ of women with PPD commit suicide..$^{10-17}$ Up to $30 \%$ of affected women still have depressive symptoms 1 year postpartum, and for some, symptoms persist for up to 11 years. ${ }^{18,19}$ Because PPD is frequently undiagnosed or unrecognized, comprehension of its potentially long-lasting detrimental effects on families and children can be obscured among policy and decision makers. ${ }^{20}$ 


\begin{tabular}{|c|c|c|c|c|c|c|}
\hline Parameter & Mean & $\begin{array}{c}\text { Standard } \\
\text { Error/N }\end{array}$ & Low & High & $\begin{array}{c}\text { Varied in PSA } \\
\text { and Distribution } \\
\text { Assumed }\end{array}$ & Reference \\
\hline \multicolumn{7}{|l|}{ Baseline EPDS distribution, \% } \\
\hline Depressive symptom range 1 & 0.0 & $\mathrm{~N}=0$ & & & & \multirow{5}{*}{45} \\
\hline Depressive symptom range 2 & 0.0 & $N=0$ & & & & \\
\hline Depressive symptom range 3 & 0.0 & $\mathrm{~N}=0$ & & & & \\
\hline Depressive symptom range 4 & 30.4 & $\mathrm{~N}=28.3^{\mathrm{a}}$ & & & & \\
\hline Depressive symptom range 5 & 69.6 & $\mathrm{~N}=64.8^{\mathrm{a}}$ & & & & \\
\hline \multicolumn{7}{|l|}{ Baseline characteristics } \\
\hline Age, years & 28 & & 18 & 40 & Uniform & \multirow{2}{*}{43} \\
\hline Weeks since birth & 16 & & 2 & 30 & Uniform & \\
\hline \multicolumn{7}{|l|}{ Efficacy (change from baseline EPDS) } \\
\hline \multicolumn{6}{|l|}{ BRX } & \multirow{8}{*}{45} \\
\hline Hour 60 (week 0.36) & -8.34 & 0.93 & -6.52 & -10.17 & Normal & \\
\hline Day 30 (week 4) & -9.75 & 1.12 & -7.55 & -11.95 & Normal & \\
\hline Week $18^{\mathrm{b}}$ & -9.75 & 1.12 & -7.55 & -11.95 & Normal & \\
\hline \multicolumn{6}{|l|}{ SSRIs } & \\
\hline Hour 60 (week 0.36c) & -0.36 & 1.36 & 2.29 & -3.02 & Normal & \\
\hline Day 30 (week 4) & -3.40 & 1.64 & -0.18 & -6.62 & Normal & \\
\hline Week 18 & -5.70 & 1.66 & -2.44 & -8.96 & Normal & \\
\hline Suicide rate, \% & & & & & & \\
\hline Depressive symptom ranges $1-3$ & 0.00 & & 0.00 & 0.00 & Uniform & \multirow{3}{*}{$15,47,49,50$} \\
\hline Depressive symptom range 4 & 0.50 & & 0.00 & 0.90 & Uniform & \\
\hline Depressive symptom range 5 & 0.50 & & 0.00 & 0.90 & Uniform & \\
\hline \multicolumn{7}{|l|}{ Health utilities } \\
\hline \multicolumn{7}{|l|}{ For mothers } \\
\hline Depressive symptom range 1 & 0.904 & 0.0102 & 0.884 & 0.924 & Beta & \multirow{5}{*}{$\begin{array}{l}\text { SF-6D utilities estimated } \\
\text { from SF- } 36 \text { responses } \\
\text { collected in pivotal } \\
\text { brexanolone trials }\end{array}$} \\
\hline Depressive symptom range 2 & 0.785 & 0.0082 & 0.769 & 0.801 & Log-normal & \\
\hline Depressive symptom range 3 & 0.671 & 0.0057 & 0.660 & 0.682 & Log-normal & \\
\hline Depressive symptom range 4 & 0.601 & 0.0067 & 0.588 & 0.614 & Log-normal & \\
\hline Depressive symptom range 5 & 0.529 & 0.0106 & 0.508 & 0.550 & Log-normal & \\
\hline \multicolumn{7}{|l|}{ For children (aged 0-4 years) } \\
\hline Depressive symptom range 1 & 0.880 & 0.0236 & 0.834 & 0.926 & Beta & \multirow{2}{*}{57} \\
\hline Depressive symptom ranges $2-5$ & 0.880 & 0.0236 & 0.834 & 0.926 & Log-normal & \\
\hline \multicolumn{7}{|l|}{ For children (aged 5-12 years) } \\
\hline Depressive symptom range 1 & 0.875 & 0.0232 & 0.829 & 0.920 & Beta & \multirow{3}{*}{52,57} \\
\hline Depressive symptom ranges 2-3 & 0.875 & 0.0232 & 0.829 & 0.920 & Log-normal & \\
\hline Depressive symptom ranges 4-5 & 0.872 & 0.0230 & 0.827 & 0.917 & Log-normal & \\
\hline \multicolumn{7}{|l|}{ For children (aged 13+ years) } \\
\hline Depressive symptom range 1 & 0.819 & 0.0513 & 0.719 & 0.920 & Beta & \multirow{3}{*}{53,58} \\
\hline Depressive symptom ranges $2-3$ & 0.819 & 0.0513 & 0.719 & 0.920 & Log-normal & \\
\hline Depressive symptom ranges 4-5 & 0.812 & 0.0510 & 0.712 & 0.912 & Log-normal & \\
\hline \multicolumn{7}{|l|}{ For partners (1 year from birth) } \\
\hline Depressive symptom range 1 & 0.837 & 0.0521 & 0.735 & 0.939 & Beta & \multirow{3}{*}{$54,58,59$} \\
\hline Depressive symptom ranges $2-3$ & 0.837 & 0.0521 & 0.735 & 0.939 & Log-normal & \\
\hline Depressive symptom ranges 4-5 & 0.826 & 0.0516 & 0.725 & 0.928 & Log-normal & \\
\hline
\end{tabular}

Depressive symptom ranges 4-5

0.826

0.725

Children exposed to maternal depression early in life may experience long-term disruptions in neural activity and heightened stress responses. ${ }^{21}$ Weak maternal bonding in infancy can critically impair child development. ${ }^{22}$ A recently published 18-year longitudinal cohort study $(\mathrm{N}=9,848)$ demonstrated that the children of mothers with persistent and severe PPD were at a higher risk of having behavioral problems at age 3.5 (odds ratio $[\mathrm{OR}]=4.84 ; 95 \%$ confidence interval $[\mathrm{CI}]=2.94$ 7.98), lower mathematical grades at age $16(\mathrm{OR}=2.65 ; 95 \%$ $\mathrm{CI}=1.26-5.57)$, and depression at age $18(\mathrm{OR}=7.44 ; 95 \%$ $\mathrm{CI}=2.89-19.11) .{ }^{19}$ Other smaller cohort studies reported similar results. ${ }^{23-28}$ Early intervention to resolve maternal 


\section{TABLE 1 Input Parameters (continued)}

\begin{tabular}{|c|c|c|c|c|c|c|}
\hline Parameter & Mean & $\begin{array}{l}\text { Standard } \\
\text { Error/N }\end{array}$ & Low & High & $\begin{array}{c}\text { Varied in PSA } \\
\text { and Distribution } \\
\text { Assumed }\end{array}$ & Reference \\
\hline \multicolumn{7}{|l|}{ Direct medical costs, $\$$} \\
\hline Cost of BRX (4.58 vials assumed) & $34,000.00$ & & & & & WAC of 7,450 per vial \\
\hline Cost of BRX administration & $4,495.00$ & $4,495.00$ & $3,596.00$ & $5,394.00$ & Gamma & $\begin{array}{l}\text { MS-DRG } 881 \text {. CMS, FY } \\
2018 \text { Hospital Inpatient PPS } \\
\text { Final Rule, based on Tables } \\
\text { 1A, 1D, and } 5 \text {. Upper and } \\
\text { lower bounds selected to } \\
\text { simulate possible adminis- } \\
\text { tration costs if population } \\
\text { were composed of entirely } \\
\text { Medicaid or privately } \\
\text { insured patients }\end{array}$ \\
\hline $\begin{array}{l}\text { Cost of SSRIs (per week) in SSRI } \\
\text { and BRX arms }\end{array}$ & 0.97 & 0.87 & 0.18 & 3.59 & Gamma & $\begin{array}{l}\text { Average mg cost of sertra- } \\
\text { line (REDBOOK) } \times \text { Zoloft } \\
\text { daily dose for MDD }(50 \mathrm{mg})\end{array}$ \\
\hline Weeks of SSRIs in SSRI and BRX arms & 26 & & 8 & 52 & Uniform & Assumption \\
\hline BRX patients who receive SSRIs, \% & 21.60 & 5.84 & 11.26 & 31.94 & Beta & 43 \\
\hline \multicolumn{7}{|l|}{ For mothers $<1$ year postpartum } \\
\hline Depressive symptom ranges $1-2$ & 56.93 & 8.47 & 40.33 & 73.53 & Gamma & \multirow{3}{*}{60} \\
\hline Depressive symptom range 3 & 69.76 & 16.29 & 45.07 & 108.92 & Gamma & \\
\hline Depressive symptom ranges 4-5 & 146.77 & 63.20 & 73.53 & 321.27 & Gamma & \\
\hline \multicolumn{7}{|l|}{ For mothers $\geq 1$ year postpartum } \\
\hline Depressive symptom ranges 1-3 & 98.69 & 1.24 & 96.26 & 101.11 & Gamma & \multirow{2}{*}{61} \\
\hline Depressive symptom ranges $4-5$ & 233.26 & 2.19 & 228.98 & 237.55 & Gamma & \\
\hline \multicolumn{7}{|l|}{ For children (aged 0-4 years) } \\
\hline Depressive symptom ranges $1-3$ & 15.14 & 0.00 & 15.14 & 15.14 & Gamma & \multirow{2}{*}{51,62} \\
\hline Depressive symptom ranges 4-5 & 18.17 & 1.93 & 15.14 & 22.72 & Gamma & \\
\hline \multicolumn{7}{|l|}{ For children (aged 5-12 years) } \\
\hline Depressive symptom ranges $1-3$ & 6.46 & 2.96 & 1.94 & 13.53 & Gamma & \multirow{2}{*}{52,63} \\
\hline Depressive symptom ranges 4-5 & 10.32 & 4.27 & 3.09 & 21.59 & Gamma & \\
\hline \multicolumn{7}{|l|}{ For children (aged 13+ years) } \\
\hline Depressive symptom ranges $1-3$ & 15.21 & 0.11 & 15.00 & 15.42 & Gamma & \multirow{2}{*}{53,61} \\
\hline Depressive symptom ranges 4-5 & 19.16 & 0.13 & 18.90 & 19.43 & Gamma & \\
\hline \multicolumn{7}{|l|}{ For partners (1 year from birth) } \\
\hline Depressive symptom ranges 1-3 & 6.46 & 0.05 & 6.37 & 6.55 & Gamma & \multirow{2}{*}{$54,59,61$} \\
\hline Depressive symptom ranges $4-5$ & 11.74 & 0.08 & 11.58 & 11.90 & Gamma & \\
\hline \multicolumn{7}{|l|}{ Model parameters } \\
\hline Time horizon & 11 years & & 4 weeks & 18 years & & 19 \\
\hline Discount rate, \% & 3.0 & & 0.0 & 6.0 & & 79 \\
\hline
\end{tabular}

Note: Outcomes were discounted at 3\% annually and varied $0 \%$ to $6 \%$ in sensitivity analysis. ${ }^{79}$

aReweighted sample size based on match-adjusted indirect comparison.

${ }^{b} B R X$ day 30 data carried forward to week 18.

cHour 60 linearly interpolated from baseline and week 4 data.

$B R X=$ brexanolone; $C M S=$ Centers for Medicare \& Medicaid Services; EPDS =Edinburgh Postnatal Depression Scale; FY=fiscal year; MDD =major depressive disorder; MS-DRG = Medicare Severity-Diagnosis Related Group; PPS = prospective payment system; PSA = probabilistic sensitivity analysis; $S F=$ short form; $S S R I=$ selective serotonin reuptake inhibitor; WAC $=$ wholesale acquisition cost.

depression can improve mother-baby bonding and limit cognitive and behavioral developmental deficiencies. ${ }^{21}$

The Edinburgh Postnatal Depression Scale (EPDS) is the most frequently used screening questionnaire for PPD; while not specifically designed for diagnosis, it is often used for this purpose..$^{29,30}$ Once diagnosed, management of patients typically follows a stepped-care approach: women with mild symptoms are generally offered low-intensity interventions, whereas women who do not respond to these treatments or have more severe symptoms or acute presentation are often offered pharmacologic treatment, alone or adjunctive to lowintensity interventions. ${ }^{31,32}$ 


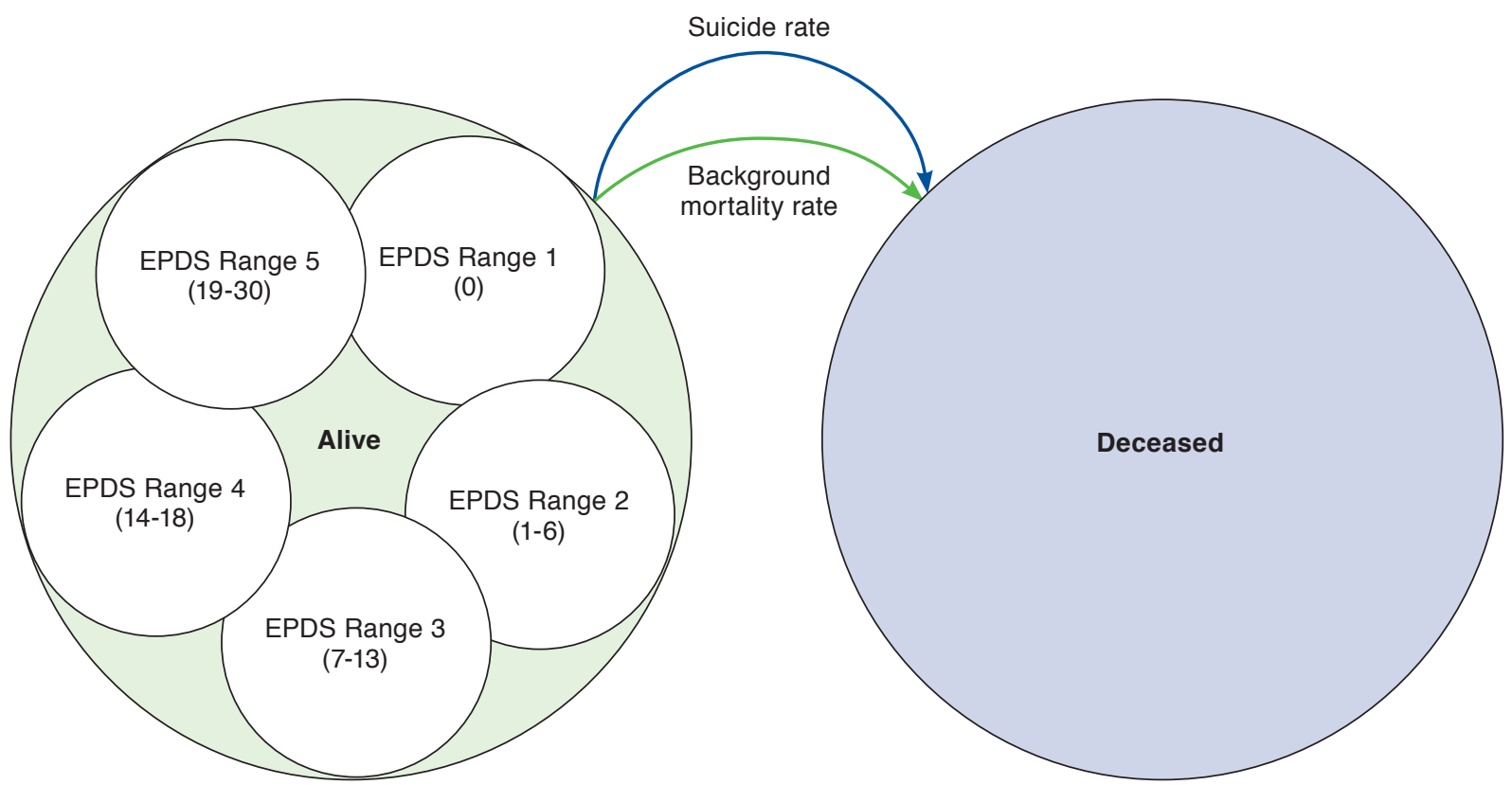

Note: The EPDS depressive symptom ranges were defined by score ranges reported in McCabe-Beane et al. (2016) ${ }^{30}$ : Range 1 (none; EPDS $=0$ ); Range 2 (minimal;

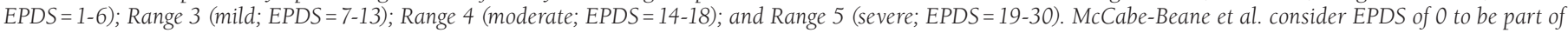
the none-to-mild group, whereas we break these into 2 separate states.

EPDS = Edinburgh Postnatal Depression Scale.

Pharmacologic treatment options include selective serotonin reuptake inhibitors (SSRIs) and other antidepressants; however, the effectiveness of these therapies for PPD has never been evaluated in robust clinical trials. ${ }^{33-35}$ A review in the Cochrane Database of Systematic Reviews of antidepressant treatment in PPD reported that relevant evidence is limited; studies had small sample sizes and a substantial risk of bias because of high dropout rates and selective reporting. ${ }^{33}$ Even when effective for PPD, SSRIs can take 6-12 weeks to achieve maximal symptom improvement, and their use may be limited by subtherapeutic dosing, poor patient adherence, and inadequate clinician follow-up. ${ }^{36-41}$

Despite the substantial unmet need in PPD, there has been no treatment approved by the U.S. Food and Drug Administration (FDA) specifically indicated for this condition until recently. In 2019, the FDA approved brexanolone injection (BRX), a neuroactive steroid chemically identical to allopregnanolone, for the treatment of PPD, under the brand name Zulresso. BRX is administered via 60-hour intravenous infusion in a single dose provided in a monitored health care setting. ${ }^{42}$ An integrated analysis of randomized placebo-controlled trials of BRX demonstrated significantly greater least-squares mean reduction in the Hamilton Depression Rating Scale total score at 60 hours compared with placebo, a reduction that was sustained for the duration of the study period (30 days). ${ }^{43}$

The objective of this study was to inform the pharmacological decision in the PPD clinical treatment cascade by evaluating the cost-effectiveness of BRX compared with conventional therapies (SSRIs) from a U.S. payer perspective. The model developed for this analysis characterizes depression in terms of the EPDS, a measure collected as a secondary endpoint in BRX trials and previously used to link maternal PPD symptoms with long-term child outcomes. ${ }^{19}$

\section{Methods}

\section{Overview of Analysis}

We used a closed-cohort state transition model to assess the cost-effectiveness of BRX compared with SSRI treatment strategies. In the base case analyses, we simulated the clinical and economic effects of these 2 strategies on women and their children over an 11-year time horizon. The base case horizon corresponded to the last observation time point for maternal EPDS scores available in Netsi et al. (2018), the most extensive study of the natural history of PPD. ${ }^{19}$ Based on these simulations, we calculated total and incremental costs from a U.S. payer perspective, total and incremental quality-adjusted 
Treatment Decision

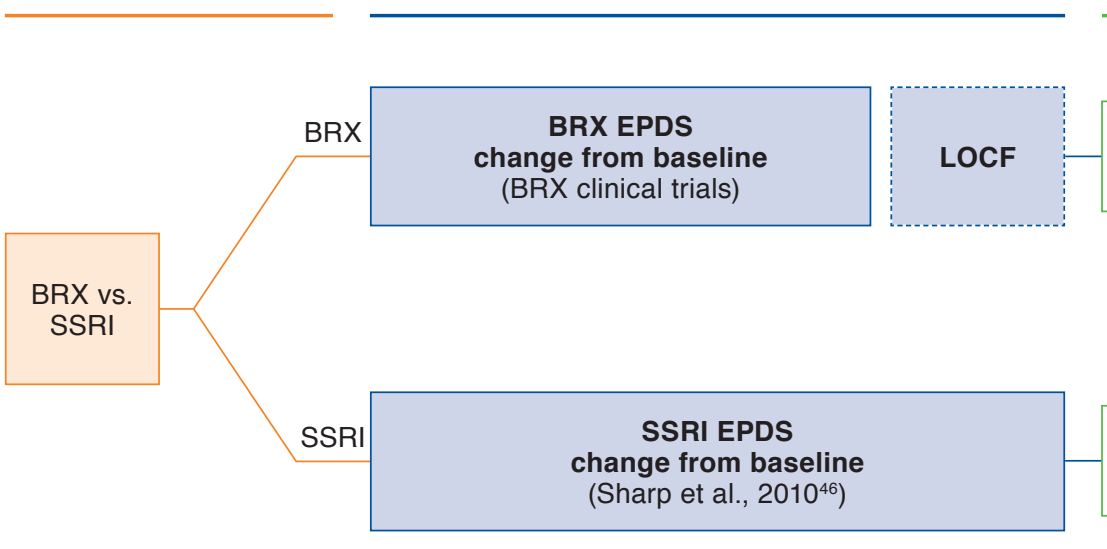

Time on treatment

(Modeled time)
Trial-Based Data

Simulated Distributions Based on Netsi et al. (2018) ${ }^{19}$

\begin{tabular}{|c|c|c|c|c|c|c|}
\hline $\begin{array}{l}\text { Treatment } \\
\text { Initiation }\end{array}$ & $\begin{array}{c}\text { Hour } \\
60\end{array}$ & $\begin{array}{c}\text { Day } \\
30\end{array}$ & $\begin{array}{c}\text { Week } \\
18^{a}\end{array}$ & $\begin{array}{c}\text { Month } \\
18^{\mathrm{b}}\end{array}$ & $\begin{array}{c}\text { Month } \\
30^{\mathrm{b}}\end{array}$ & $\begin{array}{c}\text { Year } \\
11\end{array}$ \\
\hline
\end{tabular}

aWeek 18 (month 4.5) modeled time equivalent to month 8 since birth, since the average BRX patient enrolled in the trial about 16 weeks after birth.

${ }^{b}$ Observation time points correspond to months 21 and 33, respectively, from Netsi et al. ${ }^{19}$

$B R X=$ brexanolone; $E P D S=$ Edinburgh Postnatal Depression Scale; $L O C F=$ last observation carried forward; $P P D=$ postpartum depression; SSRI = selective serotonin reuptake inhibitor.

life-years (QALYs), and the incremental cost-effectiveness ratio (ICER). Whenever possible, inputs to the model were based on analyses of individual patient data from PPD-specific studies. Model development and reporting followed best practices outlined in the Consolidated Health Economic Evaluation Reporting Standards. ${ }^{44}$

\section{Model Structure and Patient Population}

Model inputs appear in Table 1. A closed-cohort state transition model was developed in Excel 2016 (Microsoft, Redmond, WA) with 2 states for mothers: alive and deceased (Figure 1). Within the alive state, mothers were distributed by EPDS scores into 5 depressive symptom ranges, each with its own associated costs and health utilities. The EPDS depressive symptom ranges were defined by severity thresholds reported in McCabe-Beane et al. (2016) ${ }^{30}$ : Range 1 (none; 0); Range 2 (minimal; 1-6); Range 3 (mild; 7-13); Range 4 (moderate; 14-18); and Range 5 (severe; 19-30). Maternal EPDS-based health states were used to model child and partner outcomes.

The model began at the point of a PPD pharmacological treatment decision (treat with BRX or with SSRI). Cycle duration was 1 week and outcomes were assessed from 4 weeks to 18 years (when a child is assumed to be independent), with a base case time horizon of 11 years (the follow-up time for the longest EPDS-based cohort of mothers with PPD, as reported in Netsi et al.). ${ }^{19}$

The modeled patient population represented the sample followed in the BRX trials, specifically mothers who were on average 16 weeks postpartum and aged 28 years. ${ }^{43}$

\section{Efficacy: EPDS State Distribution from 0 to 18 Weeks After Beginning of Treatment}

Figure 2 summarizes projection of EPDS scores. For BRX, we estimated the proportion of women within each of the 5 symptom ranges from EPDS distributions reported in the BRX clinical trials at baseline, hour 60, and day 30, and used linear interpolation to estimate these proportions at time points between hour 60 and day $30 .{ }^{45}$ The model carried the day 30 proportions forward through week 18. Before using the BRX results, we conducted a match-adjusted indirect comparison (MAIC) to weight BRX trials patients so that their baseline characteristics matched those of patients in a randomized controlled trial of antidepressant therapy for PPD conducted by Sharp et al. (2010). ${ }^{45,46}$

For women receiving SSRI therapy, we estimated EPDS score trajectories in an analogous manner from the Sharp et al. trial, using results reported at baseline, hour 60, day 30, and at final observation (week 18). ${ }^{46}$ 
The sensitivity analysis for short-term efficacy varied EPDS distributions based on the standard errors estimated in the MAIC.

\section{Efficacy: EPDS State Distribution 18 Weeks After Beginning of Treatment}

We used results from Netsi et al. to characterize maternal EPDS trajectories 18 weeks after beginning treatment. Netsi et al. designated women with severe PPD (EPDS score of 17 or higher at 2 months postpartum) as "persistent" if their EPDS score remained 17 or higher at 8 months postpartum, or "not persistent" if their EPDS score at 8 months postpartum fell below $17 .{ }^{19}$ Based on our short-term simulation results that have already been described, we calculated the proportion of women in the BRX and SSRI treatment arms in each of these 2 categories at 18 weeks after beginning treatment (a time point corresponding to approximately 8 months postpartum and hence consistent with the Netsi et al. approach).

For the simulated cohort with EPDS scores at or exceeding 17 at 18 weeks after beginning treatment, we assumed subsequent EPDS scores had mean values equal to the means observed in the Netsi et al. "severe, persistent" subgroup at 18 months, 30 months, and 11 years after beginning treatment. For the cohort with EPDS scores below 17 at 18 weeks after beginning treatment, we assumed subsequent EPDS scores had means at these time points as observed in the Netsi et al. "severe, not persistent" subgroup.

To characterize the distribution of EPDS scores around these means, we assumed beta distributions bounded at 0 and 30 with standard deviation assumed to equal those reported by Netsi et al.

In the sensitivity analysis, we examined outcomes using the last observation carried forward (LOCF) from the MAIC after model week 18 rather than the outcome trajectory approach using Netsi et al. categorizations.

\section{Mother's Mortality}

We assumed mortality equaled the sum of the age-based background rate based on U.S. life tables and an excess suicide rate, which we assumed was higher among mothers with EPDS scores of 14 or higher. ${ }^{15,47-50}$

\section{Children's Outcomes}

Children aged 0-4 years of mothers with moderate to severe PPD were assumed to be at increased likelihood of hospitalization. ${ }^{51}$ Children aged 5-12 years of mothers with moderate to severe PPD were assumed to be at increased likelihood of experiencing behavioral issues..$^{52}$ Children aged 13-18 years of mothers with moderate to severe PPD were assumed to be at increased likelihood of depression..$^{53}$

\section{Partners' Outcomes}

Sensitivity analysis investigated the additional effect of PPD on partners. In the first simulation year, partners of mothers with moderate to severe PPD were assumed to have an elevated risk of major depressive disorder (MDD). ${ }^{54}$

\section{Health Utility}

We estimated utility for mothers from short-form 6D (SF-6D) data based on BRX clinical trial SF-36 data. ${ }^{55}$ We assumed children aged 0-4 years experience no utility loss, regardless of the maternal EPDS score. For older children, the utility loss of having a mother with moderate or severe depression has 2 components: (a) the direct effect of the mother's depression and her associated behavior, and (b) the child's elevated likelihood of experiencing depression or behavioral difficulties. Netsi et al. report data from the ALSPAC dataset using the Rutter Total Problems Scale to measure children's hyperactivity and emotional and conduct problems. The Rutter score was reported for mothers with persistent and nonpersistent severe PPD episodes. However, the Rutter scale has not been related to health utility in any publication.

Attention deficit hyperactivity disorder (ADHD) often presents with hyperactivity, as well as emotional and conduct problems, and has been related to health utility. ${ }^{56,57}$ Accordingly, ADHD was used as a proxy for behavioral difficulties. Children aged 5-12 years were assumed to have health utilities equivalent to those of children with ADHD if their mothers had moderate to severe PPD and equivalent to non-ADHD siblings if their mothers had mild, minimal, or no PPD. ${ }^{57}$ For children aged 5-12 years, the likelihood of increased behavioral problems from a study of behavioral difficulties in children of depressed mothers was used to calculate a weighted average utility for children of mothers with moderate to severe PPD compared to those with mild, minimal, or no PPD. ${ }^{52}$ Children aged 13 years and older were assumed to have health utilities equivalent to MDD "nonresponders" if their mothers had moderate to severe PPD, while children with mothers mild, minimal, or no PPD were assumed to have utility values of MDD patients who were classified as "responder remitters." ${ }^{\text {" }}$ For teenagers of mothers with PPD, the likelihood of being depressed was derived from a study of offspring depression in depressed mothers. ${ }^{53}$

In sensitivity analyses, partners who experience MDD also experience a health decrement. ${ }^{54,58,59}$ The partners of mothers with moderate to severe PPD were assumed to experience a utility weight decrement of 0.011, compared with the partners of mothers with mild, minimal, or no PPD; this was assumed to end after the first year of the simulation.

\section{Treatment Costs}

BRX treatment was assumed to cost $\$ 34,000$ (assuming an average of 4.58 vials and a wholesale acquisition cost of $\$ 7,450$ per vial), with this cost incurred during the first model cycle. We assumed BRX to be administered in a hospital setting, adding a cost of $\$ 4,495$. This estimate is based on diagnosis-related group code 881 for depressive neuroses. In clinical trials, BRX 
patients receiving antidepressants at baseline could continue treatment; reflecting the observed rate of continued antidepressant use, we assumed that $21.6 \%$ of BRX patients incurred the cost of SSRI treatment $(\$ 25.12$ over 26 weeks, or $\$ 0.97$ per week, reflecting the cost of generic sertraline).

\section{Other Direct Medical Costs}

Direct medical costs for mothers during the first year postpartum were based on a study of excess costs for privately insured, employed mothers with PPD (EPDS score $\geq 13$ ) in 3 U.S. hospitals over 11 weeks postpartum. ${ }^{60}$ Direct medical costs for mothers thereafter were based on published estimates for MDD. ${ }^{61}$

Children's direct medical costs for those aged 0-4 years were based on costs of hospitalization among infants in the United States. ${ }^{62}$ For children aged 5-12 years, costs were based on a study of the economic effect of ADHD in children and adolescents. ${ }^{63}$ For children aged 13 years and older, direct medical costs were based on published costs for MDD. ${ }^{61}$

Partners' costs were based on the economic burden of MDD and used in the sensitivity analysis. ${ }^{61}$ In the first simulation year, partners of mothers with moderate to severe PPD were assumed to have an elevated risk of depression. ${ }^{54}$ The incremental direct medical cost burden for patients with MDD was applied to a larger share of partners of mothers with MDD than those without, who were assumed to have a prevalence rate of MDD equal to that of the general adult male population in the United States. ${ }^{59}$

Costs were inflation-adjusted to 2018 U.S. dollars based on the medical care component of the Consumer Price Index. ${ }^{64}$

\section{Deterministic and Probabilistic Sensitivity Analysis}

Deterministic sensitivity analysis (DSA) varied each parameter individually and assessed the effect on the results. Probabilistic sensitivity analysis (PSA) simultaneously varied all model parameters in a Monte Carlo simulation of 1,000 iterations. Distributions for each assumption appear in Table 1. Results are presented as cost-effectiveness acceptability curves. ${ }^{65}$

\section{Scenario Analysis}

Our base case analysis includes only benefits (QALYs accrued) by mothers and their children under the assumption that from the perspective of the payer, outcomes for only these individuals matter. Our first scenario analysis broadens this perspective to include health benefits accruing to the partners of the mothers. Limiting inclusion of partner health benefits to the scenario analysis also reflects the fact that data for these health impacts are limited compared with the data for mothers and children.

Our base case analysis includes mothers represented by patients in the BRX clinical trials (i.e., patients with moderate or severe PPD [EPDS $\geq 14]$ at baseline). Because BRX administration takes place in a monitored health care setting, however, it is reasonable to assume that in the real world, initial cohorts (i.e., early adopters) will be limited to individuals with the most severe symptoms. Our second scenario analysis explored the implications of treating this cohort by limiting inclusion to mothers with severe PPD (EPDS $\geq 19)$ at baseline.

\section{Results}

At 4 weeks, 84.5\% of BRX-treated mothers were in remission (defined as having EPDS scores within ranges 1-3; i.e., EPDS scores $\leq 14)$ compared with $16.2 \%$ of SSRI-treated mothers. Corresponding proportions at 1 year and 11 years were $77.4 \%$ and $72.5 \%$ for BRX-treated mothers and $51.2 \%$ and $63.1 \%$ SSRI-treated mothers, respectively.

Table 2 presents base case costs and QALYs. Treatment costs associated with BRX averaged $\$ 38,501$, compared with $\$ 25$ for SSRIs. Direct medical costs for mothers, excluding treatment, were $\$ 65,908$ with BRX, and $\$ 73,653$ with SSRIs. BRX-treated mothers had more QALYs on average (6.230) than did SSRI-treated mothers (5.979). On average, the children of BRX-treated mothers incurred $\$ 5,602$ of direct medical costs and had 8.142 QALYs. The children of SSRI-treated mothers incurred direct medical costs of $\$ 5,800$ and had 8.106 QALYs.

Compared with SSRIs, BRX treatment costs were higher, mother and child health care costs were lower, and incremental QALY gains were 0.250 for mothers and 0.036 for children. The ICER for BRX compared with SSRIs was $\$ 106,662$.

Table 2 presents scenario analyses results. In the first alternative scenario analysis, which included partner health care costs and QALYs (i.e., impacts during the first year following the child's birth), the ICER for BRX versus SSRIs was $\$ 105,109$. The second alternative scenario analysis included only women with severe PPD (EPDS $\geq 19)$ at baseline. Largely because the maternal health benefit increases to 0.348 QALYs from its base case value of 0.250 QALYs, the ICER drops to $\$ 68,619$.

The DSA assessed the influence of each parameter on the model-projected ICER by holding all assumptions equal to their respective base case values, and then varying each assumption, one at a time over its plausible range. The Appendix (available in online article) illustrates the influence of the 20 most influential assumptions. We note that this figure does not display the full range of ICER values corresponding to alternative time horizon assumptions because (a) use of the shortest time horizon we considered (4 weeks) yields an ICER of $\$ 5,433,115$ per QALY gained and (b) extension of the horizontal axis to that value would have rendered the DSA ICER results for other assumptions difficult to discern because they span a much smaller range. Other influential assumptions include SSRI efficacy at week 18 and use of projecting long-term outcomes using LOCF after the end of the trial in place of using the Netsi et al. values (used in the base case) to project long-term outcomes. Our analysis found that use of the Netsi et al. values produced a less favorable ICER than use of LOCF. 


\begin{tabular}{|c|c|c|c|}
\hline & BRX & SSRIs & $\begin{array}{c}\text { BRX vs. } \\
\text { SSRIs }\end{array}$ \\
\hline \multicolumn{4}{|c|}{ Base case: mother (moderate and severe) and child } \\
\hline Treatment costs, $\$$ & 38,501 & 25 & 38,476 \\
\hline Mother's health care costs, $\$$ & 65,908 & 73,653 & $-7,745$ \\
\hline Child's health care costs, $\$$ & 5,602 & 5,800 & -198 \\
\hline Mother's QALYs & 6.230 & 5.979 & 0.250 \\
\hline Child's QALYs & 8.142 & 8.106 & 0.036 \\
\hline Incremental costs, \$ & & & 30,532 \\
\hline Incremental QALYs & & & 0.286 \\
\hline ICER, \$ & & & 106,662 \\
\hline \multicolumn{4}{|c|}{ Scenario 1: mother (moderate and severe), child, and partner } \\
\hline Treatment costs, $\$$ & 38,501 & 25 & 38,476 \\
\hline Mother's health care costs, $\$$ & 65,908 & 73,653 & $-7,745$ \\
\hline Child's health care costs, $\$$ & 5,602 & 5,800 & -198 \\
\hline Partner's health care costs, $\$$ & 271 & 350 & -78 \\
\hline Mother's QALYs & 6.230 & 5.979 & 0.250 \\
\hline Child's QALYs & 8.142 & 8.106 & 0.036 \\
\hline Partner's QALYs & 0.572 & 0.568 & 0.003 \\
\hline Incremental costs, \$ & & & 30,454 \\
\hline Incremental QALYs & & & 0.290 \\
\hline ICER, \$ & & & 105,109 \\
\hline \multicolumn{4}{|c|}{ Scenario 2: mother (severe) and child } \\
\hline Treatment costs, $\$$ & 38,501 & 25 & 38,476 \\
\hline Mother's health care costs, $\$$ & 66,804 & 76,949 & $-10,901$ \\
\hline Child's health care costs, $\$$ & 5,606 & 5,884 & -279 \\
\hline Mother's QALYs & 6.222 & 5.874 & 0.348 \\
\hline Child's QALYs & 8.141 & 8.091 & 0.050 \\
\hline Incremental costs, \$ & & & 27,296 \\
\hline Incremental QALYs & & & 0.398 \\
\hline ICER, \$ & & & 68,619 \\
\hline
\end{tabular}

$B R X=$ brexanolone; $I C E R=$ incremental cost-effectiveness ratio; $Q A L Y=$ qualityadjusted life-year; SSRI = selective serotonin reuptake inhibitor.

The PSA reflects results from 1,000 simulations, each of which used model assumption values drawn from independent probability distributions characterizing each assumption's set of plausible alternatives. The PSA results indicate that the cost-effectiveness of BRX is more favorable than $\$ 150,000$ per QALY with 58\% probability, and that it is more favorable than $\$ 100,000$ per QALY with 44\% probability (Figure 3).

\section{Discussion}

The effect of PPD symptoms on women and their families has prompted an increased emphasis on postpartum depression screening. ${ }^{66-68}$ However, the value of screening depends on treatment value. We found BRX to have an ICER below $\$ 150,000$ per QALY compared with SSRIs, suggesting that BRX achieves favorable cost-effectiveness in a U.S. context. ${ }^{69}$

Results were highly sensitive to the assumed efficacy of SSRIs and to the depression severity at baseline. Notably, scenario analysis indicated that the ICER was $\$ 68,619$ for women with severe PPD (EPDS $\geq 19$ ), which is estimated to be approximately 20\%-30\% of PPD cases. ${ }^{30,70}$ Because BRX is available only through a restricted program under a Risk Evaluation and Mitigation Strategy, requiring patient enrollment, a restricted distribution program, and administration in a certified health care facility with monitoring by a health care provider, real-world BRX use will likely be primarily among more severe PPD patients. ${ }^{42}$

The factor that most influenced results was the assumed duration of the treatment effect, with longer durations allowing accrual of health benefits for the mother to offset the upfront costs of BRX. A 4-week model time horizon yielded an ICER of about $\$ 5$ million per QALY, while extending the time horizon to 18 years reduced the ICER to about $\$ 60,000$ per QALY. Similarly, Ride (2018) found that extending the time horizon could substantially influence the estimated cost-effectiveness of a hypothetical PPD treatment. ${ }^{71}$

Because this model assumes a U.S. third-party payer perspective, it does not fully capture PPD's societal burden. In particular, we have no information regarding the effect of maternal PPD (as a function of EPDS - our model's health state indicator) on maternal labor market participation, maternal home production, maternal and social care, child education, and child involvement with the criminal justice system.

Related literature and general information on the effect of PPD suggests the societal costs of this condition are substantial. According to a research brief published by Mathematica, the societal cost related to perinatal mood and anxiety disorders (PMAD) was $\$ 14.2$ billion for 2017 births, or $\$ 32,000$ per case for untreated PMAD over a 6 -year period. ${ }^{72}$ The authors note that if PPD is left untreated, it can become a multigenerational issue, negatively affecting the mother and child's long-term physical, emotional, and developmental health. A U.K. analysis of the societal costs of perinatal mental health problems found that approximately $72 \%$ of the $£ 74,000$ in costs were related to adverse impacts to children, which likewise have societal spillovers. ${ }^{73}$ Additionally, Netsi et al. report lower educational attainment in PPD-affected children, an outcome that may affect productivity. ${ }^{19}$

The initial PPD episode can likewise increase involvement in crime and risky behaviors, and depress prosocial behavior. While quantification of these societal impacts is beyond the scope of the present paper, it appears that broadening this assessment to a societal perspective would make the estimated cost-effectiveness of BRX therapy more favorable. Our analysis also excluded sibling and extended family member outcomes, the broader consequences of maternal suicide for the family unit, and assumed a single birth, likely further underestimating the costs of persistent PPD for the whole family. 


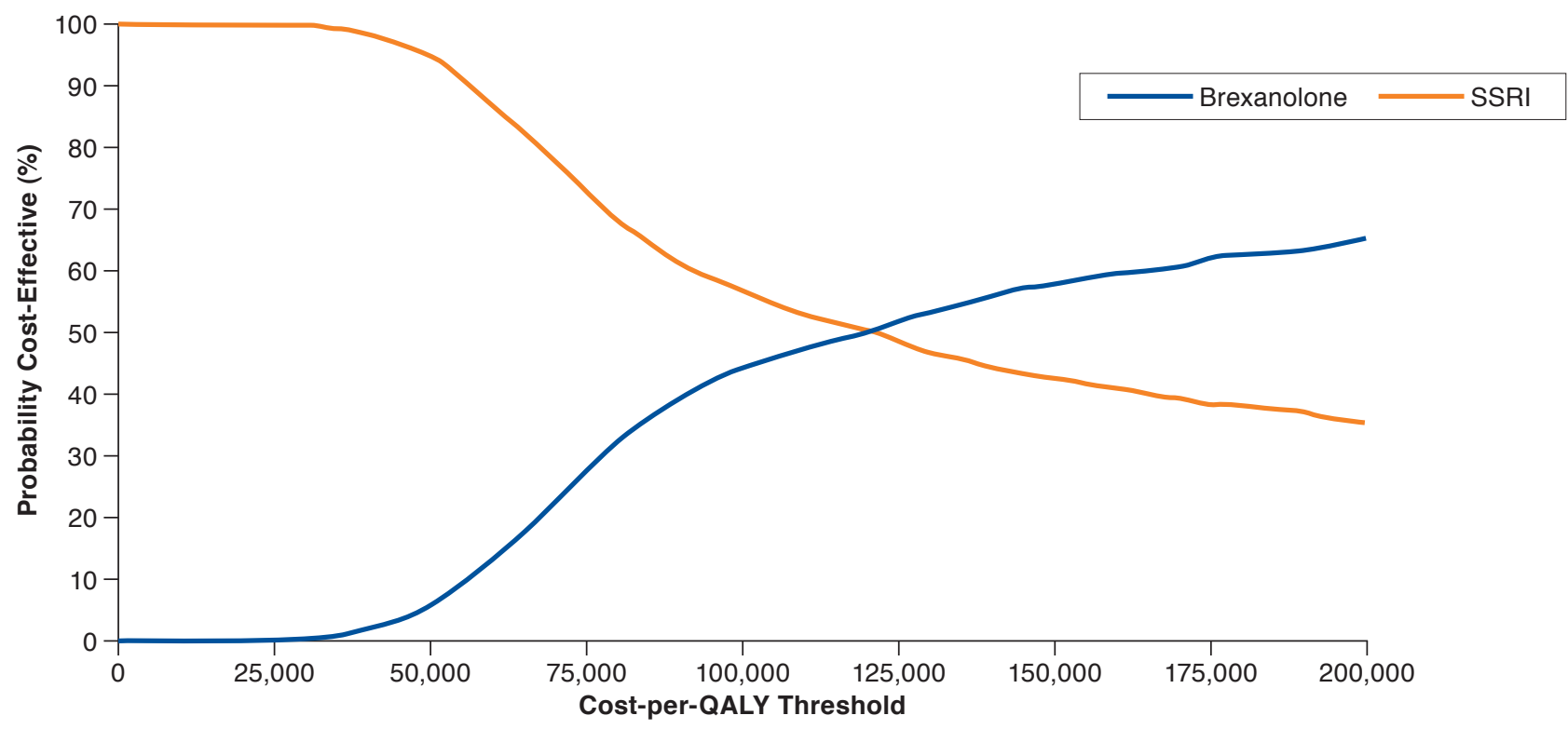

Note: This figure illustrates whether brexanolone or SSRI is more likely to be better value for money when treating PPD. The y-axis represents the proportion of simulations in which brexanolone (blue line) or SSRI (orange line) was most cost-effective. The x-axis represents the assumed value of QALY. At relatively low QALY values, SSRI treatment is more likely than brexanolone treatment to be cost-effective. Because brexanolone treatment accrues more QALYs than SSRI treatment, brexanolone is more likely to be cost-effective than SSRIs when the QALY's value is assumed to be greater (i.e., as we move to the right on the horizontal axis). In particular, brexanolone is more likely to be cost-effective than SSRIs if the QALY is assumed to have a value exceeding $\$ 120,000$.

$B R X=$ brexanolone; $Q A L Y=$ quality-adjusted life-year; SSRI = selective serotonin reuptake inhibitor.

\section{Limitations}

This analysis has some limitations to consider. A key limitation was data availability. Efficacy data were available for 4 weeks for BRX and up to 18 weeks for SSRIs. Because no model comparators had efficacy data beyond 18 weeks, we used data from Netsi et al. to project long-term outcomes over an 11-year time horizon. ${ }^{19}$ The rationale for the base case 11-year model time horizon is research suggesting that long-term maternal and child outcomes depend on the severity and duration of the PPD episode during the weeks and months following birth. 19,74,75 In addition to Netsi et al.'s 18-year longitudinal research linking persistence of maternal PPD to worse maternal and child outcomes, Campbell et al. (1995) found that the children of mothers with persistent PPD had worse outcomes than the children of mothers with shorter PPD duration. ${ }^{76}$ Petterson and Albers (2001) found that children exposed to persistent maternal depression had lower cognitive scores than children in a control group, which controlled for maternal characteristics. ${ }^{77}$ This research suggests that shorter PPD episodes during the period after birth improve long-term outcomes. The earlier the intervention to halt deprivation and improve bonding, the greater the likelihood of restoring the child's developmental trajectory. ${ }^{78}$ However, the relationship between a PPD episode and future outcomes for the affected children is not necessarily causal.

Although the Second Panel on Cost-Effectiveness in Health and Medicine recommends including family member costs and health impacts, information on these outcomes were largely unavailable for PPD populations. ${ }^{79}$ Unlike Ride, we found that effects on family members (measured in terms of their effect on the ICER) to be limited, perhaps because of imprecise measurement and a lack of PPD data, in addition to differences in model perspective. ${ }^{71}$ Limited published literature exists regarding the economic and resource burden of PPD in the United States, so we used data from the most relevant studies to fill evidence gaps.

Our analysis did not explicitly consider SSRI adherence, a factor that has shown to be a particular problem among lowincome, insured, pregnant women. ${ }^{80}$ This problem may persist into the postpartum period.

Finally, we note a series of limitations related to design of the trials we relied on for data. Because patients in both arms of the BRX clinical trials program were closely monitored in a clinical study setting with 24-hour care for 3 days, the placebo arm does not represent plausible real-world treatment for PPD in the United States, as all current care is outpatient except in the most severe cases. Our model relied on an MAIC 
that simulated a comparison between BRX and SSRIs. The randomized controlled trials for SSRIs to treat PPD generally had small sample sizes, with the exception of the Sharp et al. study. Moreover, a recent Cochrane review concluded that all of these studies have uncertain or likely bias because of a lack of representative sampling, high attrition, and selective reporting, among other factors. ${ }^{33,45}$

Given these limitations, the cost-effectiveness of pharmacological PPD treatment should be reevaluated as more data accumulate on real-world treatment effectiveness and longterm outcomes.

\section{Conclusions}

Our results suggest BRX is a cost-effective therapy compared with SSRIs for treating women with PPD in the United States with a modeled 11-year time horizon. The use of BRX to treat women with PPD for whom pharmacologic therapy is warranted could improve short- and long-term outcomes for both women and children.

\section{Authors}

ADI ELDAR-LISSAI, PhD; MARGARET E. GERBASI, PhD; and PAUL HODGKINS, PhD, Sage Therapeutics, Cambridge, Massachusetts. JOSHUA T. COHEN, PhD, Center for the Evaluation of Value and Risk in Health (CEVR), Tufts Medical Center, Boston, Massachusetts. SAMANTHA MELTZER-BRODY, MD, MPH, Department of Psychiatry, University of North Carolina School of Medicine, Chapel Hill. ELIZABETH CHERTAVIAN, MBA; JULIA C. BOND, MPH; and SCOTT J. JOHNSON, PhD, MHA, Medicus Economics, Milton, Massachusetts.

AUTHOR CORRESPONDENCE: Adi Eldar-Lissai, PhD, Sage Therapeutics, 215 First St., Cambridge, MA 02142. Tel.: 617.299.8380; E-mail: adi.eldar-lissai@sagerx.com.

\section{REFERENCES}

1. Ko JY, Rockhill KM, Tong VT, Morrow B, Farr SL. Trends in postpartum depressive symptoms-27 states, 2004, 2008, and 2012. MMWR Morb Mortal Wkly Rep. 2017;66(6):153-58.

2. Hamilton BE, Martin JA, Osterman MJK, Rossen LM. Births: provisional data for 2018. Vital Statistics Rapid Release. Report no 7. May 2019. Available at: https://www.cdc.gov/nchs/data/vsrr/vsrr-007-508.pdf. Accessed January 23, 2020

3. DeSisto CL, Kim SY, Sharma AJ. Prevalence estimates of gestational diabetes mellitus in the United States, Pregnancy Risk Assessment Monitoring System (PRAMS), 2007-2010. Prev Chronic Dis. 2014;11:E104

4. Centers for Disease Control and Prevention. Diabetes during pregnancy. June 12, 2018. Available at: https://www.cdc.gov/reproductivehealth/maternalinfanthealth/diabetes-during-pregnancy.htm. Accessed January 23, 2020.

5. Centers for Disease Control and Prevention. Data on selected pregnancy complications in the United States. February 28, 2019. Available at: https:// www.cdc.gov/reproductivehealth/maternalinfanthealth/pregnancy-complications-data.htm. Accessed January 23, 2020.

6. Roberts JM, August PA, Bakris G, et al; American College of Obstetricians and Gynecologists Task Force on Hypertension in Pregnancy. Hypertension in pregnancy. 2013. Available at: http://www.spog.org.pe/web/phocadownloadpap/HypertensioninPregnancy.pdf. Accessed January 23, 2020.

7. Callaghan WM, Kuklina EV, Berg CJ. Trends in postpartum hemorrhage: United States 1994-2006. Am J Obstet Gynecol. 2010;202(4):353.el-6.

8. American Psychiatric Association. Diagnostic and Statistical Manual of Mental Disorders. 5th ed. Washington, DC: American Psychiatric Association; 2013

9. National Institute of Mental Health. Postpartum depression facts. Available at: https://www.nimh.nih.gov/health/publications/postpartumdepression-facts/index.shtml. Accessed January 23, 2020.

10. Appleby L, Mortensen PB, Faragher EB. Suicide and other causes of mortality after post-partum psychiatric admission. Br J Psychiatry. 1998;173:209-11.

11. Moses-Kolko EL, Hipwell AE. First-onset postpartum psychiatric disorders portend high 1-year unnatural-cause mortality risk. Am J Psychiatry. 2016;173(6):559-61

12. Bodnar-Deren S, Klipstein K, Fersh M, Shemesh E, Howell EA. Suicidal ideation during the postpartum period. J Womens Health (Larchmt). 2016; 25(12):1219-24.

13. Gressier F, Guillard V, Cazas O, et al. Risk factors for suicide attempt in pregnancy and the postpartum period in women with serious mental illnesses. J Psychiatr Res. 2017;84:284-91.

14. Palladino CL, Singh V, Campbell J, Flynn H, Gold KJ. Homicide and suicide during the perinatal period: findings from the National Violent Death Reporting System. Obstet Gynecol. 2011;118(5):1056-63.

15. Lindahl V, Pearson JL, Colpe L. Prevalence of suicidality during pregnancy and the postpartum. Arch Womens Ment Health. 2005;8(2):77-87.

16. Review to Action. Report from nine maternal mortality review committees. 2018. Available at: https://reviewtoaction.org/sites/default/files/ national-portal-material/Report\%20from\%20Nine\%20MMRCs\%20final_0. pdf. Accessed January 23, 2020.

17. Mangla K, Hoffman MC, Trumpff C, O'Grady S, Monk C. Maternal self-harm deaths: an unrecognized and preventable outcome. Am J Obstet Gynecol. 2019;221(4):295-303.

18. Vliegen N, Casalin S, Luyten P. The course of postpartum depression: a review of longitudinal studies. Harv Rev Psychiatry. 2014;22(1):1-22.

19. Netsi E, Pearson RM, Murray L, Cooper P, Craske MG, Stein A. Association of persistent and severe postnatal depression with child outcomes. JAMA Psychiatry. 2018;75(3):247-53. 
20. Cox EQ, Sowa NA, Meltzer-Brody SE, Gaynes BN. The perinatal depression treatment cascade: baby steps toward improving outcomes. J Clin Psychiatry. 2016;77(9):1189-200.

21. Center on the Developing Child at Harvard University. Maternal depression can undermine the development of young children. Working paper 8 . 2009. Available at: https://developingchild.harvard.edu/resources/maternaldepression-can-undermine-the-development-of-young-children. Accessed January 23, 2020

22. Behrendt HF, Scharke W, Herpertz-Dahlmann B, Konrad K, Firk C. Like mother, like child? Maternal determinants of children's early social-emotional development. Infant Ment Health J. 2019;40(2):234-47.

23. Siqueira Barcelos R, da Silva dos Santos I, Matijasevich A, Anselmi L, Celso Barros F. Maternal depression is associated with injuries in children aged 2-4 years: the Pelotas 2004 Birth Cohort. Inj Prev. 2018;25(3):222-27.

24. Mulraney M, Giallo R, Efron D, Brown S, Nicholson J, Sciberras E. Maternal postnatal mental health and offspring symptoms of ADHD at 8-9 years: pathways via parenting behavior. Eur Child Adolesc Psychiatry. 2018;28(7):923-32

25. Stein A, Pearson RM, Goodman SH, et al. Effects of perinatal mental disorders on the fetus and child. Lancet. 2014;384(9956):1800-19.

26. Sanger C, Iles JE, Andrew CS, Ramchandani PG. Associations between postnatal maternal depression and psychological outcomes in adolescent offspring: a systematic review. Arch Womens Ment Health. 2015;18(2):147-62.

27. Murray L, Arteche A, Fearon P, Halligan S, Croudace T, Cooper P. The effects of maternal postnatal depression and child sex on academic performance at age 16 years: a developmental approach. J Child Psychol Psychiatry. 2010;51(10):1150-59.

28. Moore Simas TA, Huang MY, Patton C, et al. The humanistic burden of postpartum depression: a systematic literature review. Curr Med Res Opin. 2018;35(3):383-93

29. Howard LM, Molyneaux E, Dennis C, Rochat T, Stein A, Milgrom J. Non-psychotic mental disorders in the perinatal period. Lancet. 2014; 384:1775-88.

30. McCabe-Beane JE, Segre LS, Perkhounkova Y, Stuart S, O'Hara MW. The identification of severity ranges for the Edinburgh Postnatal Depression Scale. J Reprod Infant Psychol. 2016;34(3):293-303.

31. Meltzer-Brody S, Howard LM, Bergink V, et al. Postpartum psychiatric disorders. Nat Rev Dis Primers. 2018;4:18022.

32. American Psychiatric Association. Practice guideline for the treatment of patients with major depressive disorder. 3rd ed. October 2010. Available at: https://psychiatryonline.org/pb/assets/raw/sitewide/practice_guidelines/ guidelines/mdd.pdf. Accessed January 23, 2020.

33. Molyneaux E, Howard LM, McGeown HR, Karia AM, Trevillion K. Antidepressant treatment for postnatal depression. Cochrane Database Syst Rev. 2014;(9):CD002018

34. di Scalea TL, Wisner KL. Pharmacotherapy of postpartum depression. Expert Opin Pharmacother. 2009;10(16):2593-607.

35. Kim DR, Epperson CN, Weiss AR, Wisner KL. Pharmacotherapy of postpartum depression: an update. Expert Opin Pharmacother. 2014;15(9):1223-34.

36. Frazer A, Benmansour S. Delayed pharmacological effects of antidepressants. Mol Psychiatr. 2002;7(Suppl 1):S23-28.

37. Bushnell GA, Sturmer T, Swanson SA, et al. Dosing of selective serotonin reuptake inhibitors among children and adults before and after the FDA black-box warning. Psychiatr Serv. 2016;67(3):302-29.

38. Kelly K, Posternak M, Alpert JE. Toward achieving optimal response: understanding and managing antidepressant side effects. Dialogues Clin Neurosci. 2008;10:409-18.

39. Lingam R, Scott J. Treatment non-adherence in affective disorders. Acta Psychiatr Scand. 2002;105(3):164-72.
40. Byatt N, Simas TA, Lundquist RS, Johnson JV, Ziedonis DM. Strategies for improving perinatal depression treatment in North American outpatient obstetric settings. J Psychosom Obstet Gynaecol. 2012;33(4):143-61.

41. Jones LE, Turvey C, Carney-Doebbeling C. Inadequate follow-up care for depression and its impact on antidepressant treatment duration among veterans with and without diabetes mellitus in the Veterans Health Administration. Gen Hosp Psychiatry. 2006;28(6):465-74.

42. Zulresso (brexanolone) injection, for intravenous use. Sage Therapeutics. March 2019. Available at: https://www.accessdata.fda.gov/drugsatfda_docs/ label/2019/211371lbl.pdf. Accessed January 23, 2020.

43. Meltzer-Brody S, Colquhoun H, Riesenberg R, et al. Brexanolone injection in postpartum depression: two multicentre, double-blind, randomised, placebo-controlled, phase 3 trials. Lancet. 2018;392(10152):1058-70.

44. Husereau D, Drummond M, Petrou S, et al; CHEERS Task Force. Consolidated Health Economic Evaluation Reporting Standards (CHEERS) statement. BMJ. 2013;346:f1049.

45. Cooper M, Kilvert H, Hodgkins P, Roskell N, Eldar-Lissai A. Using matching-adjusted indirect comparisons and network meta-analyses to compare efficacy of brexanolone injection with selective serotonin reuptake inhibitors for treating postpartum depression. CNS Drugs. 2019;33(10):1039-52.

46. Sharp DJ, Chew-Graham C, Tylee A, et al. A pragmatic randomised controlled trial to compare antidepressants with a community-based psychosocial intervention for the treatment of women with postnatal depression: the RESPOND trial. Health Technol Assess. 2010;14(43):1-53.

47. Wilkinson A, Anderson S, Wheeler SB. Screening for and treating postpartum depression and psychosis: a cost-effectiveness analysis. Matern Child Health J. 2017;21(4):903-14

48. Arias E, Heron M, Xu J. United States life tables, 2014. Natl Vital Stat Rep. 2017;66(4):1-64.

49. Appleby L, Turnbull, G. Parasuicide in the first postnatal year. Psychol Med. 1995;25(5):1087-90.

50. Appleby L, Mortensen P, Faragher EB. Suicide and other causes of mortality after postpartum psychiatric admission. Brit J Psychiatry. 1998;173(3):209-11.

51. Farr SL, Dietz PM, Rizzo JH, et al. Health care utilisation in the first year of life among infants of mothers with perinatal depression or anxiety. Paediatr Perinat Epidemiol. 2013;27(1):81-88.

52. Woolhouse H, Gartland D, Mensah F, Giallo R, Brown S. Maternal depression from pregnancy to 4 years postpartum and emotional/behavioural difficulties in children: results from a prospective pregnancy cohort study. Arch Womens Ment Healt. 2016;19(1):141-51.

53. Pearson RM, Evans J, Kounali D, et al. Maternal depression during pregnancy and the postnatal period: risks and possible mechanisms for offspring depression at age 18 years. JAMA Psychiatry. 2013;70(12):1312-19.

54. Kerstis B, Aarts C, Tillman C, et al. Association between parental depressive symptoms and impaired bonding with the infant. Arch Womens Ment Health. 2016;19(1):87-94.

55. Brazier J, Roberts J, Deverill M. The estimation of a preference-based measure of health from the SF-36. J Health Econ. 2002;21(2):271-92.

56. Biederman J, Newcorn J, Sprich S. Comorbidity of attention deficit hyperactivity disorder with conduct, depressive, anxiety, and other disorders. Am J Psychiatry. 1991;148:564-77.

57. Peasgood T, Bhardwaj A, Biggs K, et al. The impact of ADHD on the health and well-being of ADHD children and their siblings. Eur Child Adolesc Psychiatry. 2016;25(11):1217-31.

58. Sapin C, Fantino B, Nowicki ML, Kind P. Usefulness of EQ-5D in assessing health status in primary care patients with major depressive disorder. Health Qual Life Outcomes. 2004;2(1):20.

59. National Institute of Mental Health. Major depression. Updated February 2019. Available at: https://www.nimh.nih.gov/health/statistics/major-depression.shtml. Accessed February 24, 2020. 
60. Dagher RK, McGovern PM, Dowd BE, Gjerdingen DK. Postpartum depression and health services expenditures among employed women. J Occup Environ Med. 2012;54(2):210-15.

61. Greenberg PE, Fournier AA, Sisitsky T, Pike CT, Kessler RC. The economic burden of adults with major depressive disorder in the United States (2005 and 2010). J Clin Psychiatry. 2015;76(2):155-62.

62. Witt WP, Weiss AJ, Elixhauser A. Overview of hospital stays for children in the United States, 2012. Heathcare Cost and Utilization Project (H-CUP). Statistical Brief \#187. December 2014. Available at: https://www.hcup-us. ahrq.gov/reports/statbriefs/sbl87-Hospital-Stays-Children-2012.jsp. Accessed February 24, 2020

63. Pelham WE, Foster EM, Robb JA. The economic impact of attentiondeficit/hyperactivity disorder in children and adolescents. J Pediatr Psychol. 2007;32(6):711-27.

64. U.S. Bureau of Labor Statistics; Federal Reserve Bank of St. Louis. Consumer price index for all urban consumers: medical care in U.S. city average. Available at: https://fred.stlouisfed.org/series/CPIMEDSL. Accessed January 23, 2020.

65. Fenwick E, Byford S. A guide to cost-effectiveness acceptability curves Br J Psychiatry. 2005;187:106-08.

66. O'Connor E, Rossom RC, Henninger M, Groom HC, Burda BU. Primary care screening for and treatment of depression in pregnant and postpartum women: evidence report and systematic review for the US Preventive Services Task Force. JAMA. 2016;315(4):388-406.

67. Siu AL, Bibbins-Domingo K, Grossman DC, et al. Screening for depression in adults: US Preventive Services Task Force recommendation statement. JAMA. 2016;315(4):380-87.

68. Alexander JL. Quest for timely detection and treatment of women with depression. J Manag Care Pharm. 2007;13(9 Supp A):3-11. Available at: https://www.jmcp.org/doi/pdf/10.18553/jmcp.2007.13.issue-s9-a.

69. Neumann PJ, Cohen JT, Weinstein MC. Updating cost-effectivenessthe curious resilience of the \$50,000-per-QALY threshold. N Engl J Med. 2014;371(9):796-97.

70. Putnam KT, Wilcox M, Robertson-Blackmore E, et al. Clinical phenotypes of perinatal depression and time of symptom onset: analysis of data from an international consortium. Lancet Psychiatry. 2017;4(6):477-85.
71. Ride J. Setting the boundaries for economic evaluation: investigating time horizon and family effects in the case of postnatal depression. Value Health. 2018;21(5):573-80.

72. Luca DL, Garlow N, Staatz C, Margiotta C, Zivin K. Societal costs of untreated perinatal mood and anxiety disorders in the United States. Mathematica Policy Research. April 2019. Available at: https://www.mathematica-mpr.com/download-media?MediaItemId=\{E24EE558-B67B-4BF680D0-3BC75DB12EB6\}. Accessed January 23, 2020.

73. Bauer A, Parsonage M, Knapp M, Iemmi V, Adelaja B. Costs of perinatal mental health problems. Centre for Mental Health. December 3, 2019. Available at: https://www.centreformentalhealth.org.uk/publications/costsof-perinatal-mental-health-problems. Accessed January 23, 2020.

74. Brennan PA, Hammen C, Andersen MJ, Bor W, Najman JM, Williams GM. Chronicity, severity, and timing of maternal depressive symptoms: relationships with child outcomes at age 5. Dev Psychol. 2000;36:759-66.

75. Halligan SL, Murray L, Martins C, Cooper PJ. Maternal depression and psychiatric outcomes in adolescent offspring: a 13-year longitudinal study. J Affect Disord. 2007;97:145-54.

76. Campbell SB, Cohn JF, Meyers T. Depression in first-time mothers: mother-infant interaction and depression chronicity. Dev Psychol. 1995;31(3):349-57.

77. Petterson SM, Albers AB. Effects of poverty and maternal depression on early child development. Child Dev. 2001;72(6):1794-813.

78. Conti G, Heckman J. The developmental approach to child and adult health. Pediatrics. 2013;131:S133-41.

79. Sanders GD, Neumann PJ, Basu A, et al. Recommendations for conduct, methodological practices, and reporting of cost-effectiveness analyses: second panel on cost-effectiveness in health and medicine. JAMA. 2016;316(10):1093-103

80. Wu J, Davis-Ajami ML. Antidepressant treatment persistence in lowincome, insured pregnant women. J Manag Care Pharm. 2014;20(6):631-37. Available at: https://www.jmcp.org/doi/10.18553/jmcp.2014.20.6.631.

81. America's Health Insurance Plans. National comparisons of commercial and Medicare fee-for service payments to hospitals. February 2016. Available at: https://www.ahip.org/wp-content/uploads/2016/02/ HospitalPriceComparison_2.10.16.pdf. Accessed January 23, 2020. 
SSRIs change from baseline - week $18 \Delta$ from BL Long-term outcomes ( $0=$ LOCF, $1=$ Netsi Severe Grps)

$B R X$ change from baseline - day $30 \Delta$ from BL Discount rate (annual)

Suicide Rate - health State 5 (19-30) Health utility (child, 0-4 years) - health State 5 (19-30) Health utility (child, 0-4 years) - health State 3 (7-13) Stakeholder scenario (mother only vs. mother + child)

Health utility (mother) - health State 5 (19-30) Health utility (child, 5-12 years) - health State 5 (19-30) Health utility (child, 5-12 years) - health State 2 (1-6) Health utility (child, 0-4 years) - health State 2 (1-6) Suicide Rate - health State 4 (14-18) Health care costs excluding drug/CBT (mother, $<1$ year pp) - all states SSRIs change from baseline - day $30 \Delta$ from BL Health utility (child, 0-4 years) - health state 4 (14-18) Health care costs excluding drug/CBT (mother, $<1$ year pp) - health State 5 (19-30) Health utility (mother) - health State 2 (1-6) Weeks since birth at BL
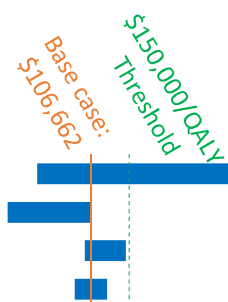

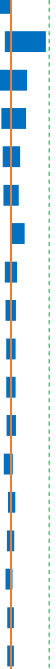

200,000

400,000

ICER, \$

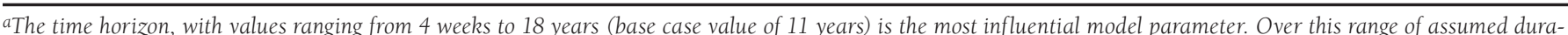

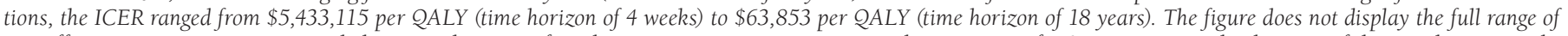

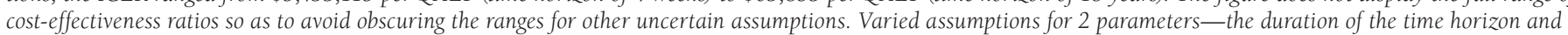
SSRI change from baseline to week 18 - crossed the \$150,000 ICER threshold.

$B L=$ baseline; $B R X=$ brexanolone; $C B T=$ cognitive behavioral therapy; ICER = incremental cost-effectiveness ratio; LOCF =last observation carried forward; pP = postpartum; QALY= quality-adjusted life-year; SSRI= selective serotonin reuptake inhibitor. 